\title{
Validity and reliability of methods for the detection of secondary caries around amalgam restorations in primary teeth
}

\section{Mariana Minatel Braga(a) Ana Paula Sturion Chiarotti(b) José Carlos Pettorossi Imparato(c) Fausto Medeiros Mendes ${ }^{(c)}$}

(a) PhD; (c) PhD, Assistant Professor - School of Dentistry, University of São Paulo, São Paulo, SP, Brazil.

(b) MSc, Hermínio Ometto University Center, University of Araras (Uniararas), Araras, SP, Brazil.

\author{
Corresponding author: \\ Mariana Minatel Braga \\ Departamento de Ortodontia e \\ Odontopediatria \\ Faculdade de Odontologia da USP \\ Av. Prof. Lineu Prestes, 2227 \\ CEP: 05508-000 \\ São Paulo - SP - Brazil \\ E-mail:mmbraga@usp.br
}

Received for publication on Sep 18, 2008 Accepted for publication on Dec 18, 2008

\begin{abstract}
Secondary caries has been reported as the main reason for restoration replacement. The aim of this in vitro study was to evaluate the performance of different methods - visual inspection, laser fluorescence (DIAGNOdent), radiography and tactile examination - for secondary caries detection in primary molars restored with amalgam. Fifty-four primary molars were photographed and 73 suspect sites adjacent to amalgam restorations were selected. Two examiners evaluated independently these sites using all methods. Agreement between examiners was assessed by the Kappa test. To validate the methods, a caries-detector dye was used after restoration removal. The best cut-off points for the sample were found by a Receiver Operator Characteristic (ROC) analysis, and the area under the ROC curve (Az), and the sensitivity, specificity and accuracy of the methods were calculated for enamel (D2) and dentine (D3) thresholds. These parameters were found for each method and then compared by the McNemar test. The tactile examination and visual inspection presented the highest inter-examiner agreement for the D2 and D3 thresholds, respectively. The visual inspection also showed better performance than the other methods for both thresholds $(\mathrm{Az}=0.861$ and $\mathrm{Az}=0.841$, respectively). In conclusion, the visual inspection presented the best performance for detecting enamel and dentin secondary caries in primary teeth restored with amalgam.
\end{abstract}

Descriptors: Dental caries; Dental amalgam; Diagnosis; Primary tooth.

\section{Introduction}

The replacement of restorations has been a frequent approach among clinicians, and secondary caries has been the major reason for explaining that. ${ }^{1}$ Since operative treatment is adopted with no control of the etiologic factors of dental caries, new caries lesions can develop around restorations, leading to the re-restoration of teeth. ${ }^{2}$

Furthermore, secondary caries detection is not a simple task for clinicians. In general, its detection at early stages is difficult. ${ }^{3}$ Discoloration next to the restoration or ditched amalgam margins are not predictive of secondary caries. ${ }^{4}$ These doubts can lead clinicians to making wrong decisions concerning the replacement of restorations.

Therefore, the accurate detection of secondary caries lesions is extremely important. The methods commonly used for this purpose have been the visual inspection, the tactile examination using a sharp probe 
and bitewing radiographs. ${ }^{1,4-6}$ Nevertheless, objective methods have also been studied in order to improve detection, and some perspectives in detection and quantification of secondary caries have been raised. ${ }^{5-8}$

Laser fluorescence (LF) is a recently proposed option for caries detection. DIAGNOdent (KaVo, Biberach, Germany) is a LF-based device, capable of distinguishing caries lesions from sound tissues based on the difference of fluorescence exhibited by the two different structures when exposed to a red and infrared spectrum. The fluorescence is transformed into a numerical scale, and the deeper the lesion, the higher the value displayed by the device. ${ }^{9}$

Even though the use of LF has been extensively studied for the detection of occlusal caries, ${ }^{10}$ few studies have been conducted about its use in the detection of secondary caries. Besides, these studies were performed in permanent teeth. , $^{6,11,12}$ Since permanent teeth are significantly different from primary teeth, ${ }^{13}$ the method should also be tested and validated in primary teeth.

Therefore, the aim of this in vitro study was to evaluate the performance of four different methods - visual inspection, LF, radiography and tactile examination - in detecting secondary caries in primary molars restored with amalgam.

\section{Material and Methods Ethical concern and sample preparation}

The experimental protocol was approved by the Local Ethics Committee. Fifty-four primary extracted or exfoliated molars presenting the occlusal surface restored with amalgam were donated by a bank of human teeth and had their surfaces photographed. One or two suspected sites were selected adjacent to the restorations $(\mathrm{n}=73)$. The specimens were cleaned with a toothbrush with pumice/water slurry and stored in saline solution until the examinations.

\section{Caries detection methods}

All selected sites were assessed by two examiners, using four different methods for caries detection: visual inspection, LF, radiography and tactile examination. The examiners were previously trained and performed the evaluations independently, unaware of each other's results.

\section{Visual inspection}

The teeth were positioned about $30 \mathrm{~cm}$ far from the examiner's eyes and air dried with a 3 -in- 1 syringe. The examiners judged the teeth according the scoring system: ${ }^{14}$

0 . No or slight change in enamel translucency after prolonged air drying ( $>5 \mathrm{~s}$ )

1. Opacity or discoloration hardly visible on wet surface, but distinctly visible after air drying

2. Opacity or discoloration distinctly visible without air drying

3. Localized enamel breakdown in opaque or discolored enamel

4. Cavitation in opaque or discolored enamel exposing the dentine

\section{LF}

An LF device (DIAGNOdent) was used. The device was calibrated against a ceramic standard and then re-calibrated after every $10^{\text {th }}$ tooth. Tooth was air-dried for $3 \mathrm{~s},{ }^{15}$ and the tip A was placed on a previously selected site and rotated around a vertical axis. The LF was calibrated on the center of the buccal surface of every tooth prior to examination of the suspected site. Three measurements were performed in each site. Peak values were registered and the mean value was calculated.

\section{Radiography}

Groups of three teeth each were fixed on an Xray film placed in a bitewing holder and radiographs were taken. The aim of this procedure was to obtain radiographs comparable to typical bitewings. The focus-to-film distance was $40 \mathrm{~cm}$.

The Spectro $70 \mathrm{X}$ unit (Dabi Atlante, Ribeirão Preto, SP, Brazil) and Kodak Ektaspeed films were used at $70 \mathrm{kV}$ and $8 \mathrm{~mA}$, with an exposure time of 0.3 seconds. The radiographs were processed in a standardized manner in order to obtain an acceptable contrast.

The radiographs were examined on a backlit screen, without magnification. For the evaluation, the examiners used the following criteria: ${ }^{7}$

1. definitely not caries 
2. probably not caries

3. questionable

4. probably caries

5. definitely caries

\section{Tactile examination}

The tactile examination was performed by probing gently the suspected site with a blunt explorer probe to avoid damage to the dental tissues. Additionaly, this examination was the last one to be performed in order to avoid interference in the results of the other methods in case of any damage.

The examiners evaluated the teeth regarding the presence of ditches and presence of softened dental tissue, using the following scores: ${ }^{6}$

0. no ditches

1. ditches hardly visible

2. ditches visible $(<0.2 \mathrm{~mm})$

3. ditches visible $(>0.2 \mathrm{~mm})$

\section{Validation}

The restorative material was removed carefully by one of the examiners using a tungsten carbide bur in a high-speed hand-piece under water refrigeration. Any contact with the cavity walls and margins was avoided. The remnants of restorative material in the cavity were removed using a sharp excavator.

Then a caries-detector dye (Vide Cárie - Inodon, Porto Alegre, RS, Brazil) was applied. The dye was applied in the cavities using a small brush, following the manufacturer's instructions. It was removed after 10 seconds by a 5 -second water spray and airdried for 5 seconds.

The suspected sites were classified according to the color of the stained tissues by both examiners and scored following a scale adapted from Yazici et al. ${ }^{16}$ (2005):

0 . white (sound)

1. red (carious) - limited to the enamel

2. red (carious) - involving dentine

\section{Statistical analysis}

A Receiver Operator Characteristic (ROC) analysis was performed for enamel (D2) and dentine (D3) thresholds, and the area under the ROC curve $(\mathrm{Az})$ and the best cut-off points were obtained. Us- ing these cut-off points, sensitivity, specificity and accuracy (percentage of correct diagnosis in sound and decayed teeth) of each method were calculated at each threshold. The McNemar test was used to assess statistically significant differences between the methods.

The inter-examiner reproducibility was assessed by Kappa coefficients, considering D2 and D3 thresholds. The level of significance for all the tests was $\mathrm{p}<0.05$.

\section{Results}

The Az for the visual inspection was higher than those for the other methods, at both thresholds $(\mathrm{D} 2=0.86 ; \mathrm{D} 3=0.84)$. At the D2 threshold, LF and tactile examination did not show statistically significant differences in Az. The same was observed between tactile examination and radiography, which presented the worst performances in enamel. At the D3 threshold, except for visual inspection, all the other methods showed similar Az values (Table 1).

The best cut-off points found for the sample are presented in Table 1. Considering sensitivity, the visual inspection also showed the highest values at the D2 threshold, and values similar to those presented by the tactile examination at the D3 threshold. Radiography presented the lowest sensitivity at both depths (Table 1).

No significant differences were observed in specificity for all the tested methods at both thresholds, except for the tactile examination, which showed lower specificity than the other methods at the D3 threshold (Table 1).

The visual inspection and the radiography showed, respectively, the highest and the lowest accuracy in detecting enamel secondary caries. All methods presented similar accuracies at the dentine thresholds. The visual inspection showed higher accuracy at the D2 threshold than at the D3 threshold. The other methods presented higher accuracy at the D3 threshold than at the D2 threshold.

At the D2 threshold, the inter-examiner reproducibility was higher for the tactile examination (0.86). At the D3 threshold, the highest inter-examiner agreement was found for the visual inspection (0.88). Radiography presented the lowest reproduc- 


\begin{tabular}{|c|c|c|c|c|c|c|}
\hline \multirow{8}{*}{$\begin{array}{r}\text { Table } 1 \text { - Sensitivity, } \\
\text { specificity and accuracy of } \\
\text { the laser fluorescence, visual } \\
\text { inspection, radiography and } \\
\text { tactile examination methods } \\
\text { for detecting secondary caries } \\
\text { lesions in primary teeth at } \\
\text { enamel (D2) and dentine } \\
\text { (D3) thresholds, and their } \\
\text { performance expressed as the } \\
\text { area under the ROC curve (Az). }\end{array}$} & Method & Best cut-off point & $A_{z}$ & Sensitivity & Specificity & Accuracy \\
\hline & \multicolumn{6}{|c|}{ Enamel (D2) } \\
\hline & Laser Fluorescence & 1 & $0.73 a$ & $0.58 a$ & $0.84 a$ & $0.65 a$ \\
\hline & Visual Inspection & 0 & $0.86 b$ & $0.78 \mathrm{~b}$ & $0.86 a$ & $0.80 \mathrm{~b}$ \\
\hline & Tactile Examination & 2 & $0.70 a, c$ & $0.41 \mathrm{c}$ & $0.93 a$ & $0.56 a$ \\
\hline & Radiography & 2 & $0.59 c$ & $0.27 d$ & $0.90 a$ & $0.46 c$ \\
\hline & \multicolumn{6}{|c|}{ Dentine (D3) } \\
\hline & Laser Fluorescence & 1.6 & $0.68 a$ & $0.56 a$ & $0.84 a$ & $0.69 a$ \\
\hline & Visual Inspection & 1 & $0.84 \mathrm{~b}$ & $0.75 b$ & $0.80 a$ & $0.77 a$ \\
\hline & Tactile Examination & 1 & $0.75 a$ & $0.74 \mathrm{~b}$ & $0.62 b$ & $0.68 a$ \\
\hline & Radiography & 1 & $0.65 a$ & $0.48 a$ & $0.72 a$ & $0.59 a$ \\
\hline
\end{tabular}

Different letters in the same column express statistically significant differences.

ibility at the enamel (0.48) and dentine thresholds (0.55) (Table 2).

\section{Discussion}

Our study aimed at testing the conventional methods, as well as the LF method, for detecting secondary caries lesions around amalgam restorations in primary teeth. Although LF is an objective method, the readings can be influenced by the presence of calculus and dental plaque, ${ }^{9,15,17}$ time of drying, ${ }^{15,17}$ the professional's experience and training ${ }^{18,19}$ and storage of the specimens for in vitro studies. ${ }^{20}$ Nevertheless, when cut-off points are adjusted for each examination separately, no significant differences have been found. ${ }^{21}$

The cut-off points will determine a limit between health and disease. Low cut-off points for LF have been related to high sensitivity values. ${ }^{17,22}$

In vitro studies generally have found lower cutoff points than in vivo studies. This fact is probably related to time and conditions of storage of the specimens. ${ }^{20,23}$ Previous studies on secondary caries detection have also shown the difference mentioned above between cut-off points obtained by in vitro and in vivo studies. This difference has been reported as being around 10 arbitrary units. ${ }^{7,11}$

In our study, the observed cut-off points were lower than those found in previous studies, both at the enamel and dentine thresholds. ${ }^{711}$ Considering that the teeth used in our study were obtained from a bank of human teeth, and that it was impossible to
Table 2 - Inter-examiner agreement for the laser fluorescence, visual examination, radiography and tactile examination methods for detecting secondary caries lesions at the enamel and dentine thresholds.

\begin{tabular}{l|c|c}
\hline \multirow{2}{*}{\multicolumn{1}{c|}{ Method }} & \multicolumn{2}{|c}{ Inter-examiner agreement } \\
\cline { 2 - 3 } & Enamel & Dentine \\
\hline Laser fluorescence & 0.66 & 0.79 \\
\hline Visual inspection & 0.71 & 0.88 \\
\hline Tactile examination & 0.86 & 0.69 \\
\hline Radiography & 0.48 & 0.55 \\
\hline
\end{tabular}

determine the storage time precisely, we assumed that the low cut-off values may have been a result of that.

Regarding the cut-off points for the conventional methods tested, an inversion was observed between the cut-off points found for radiography and for the tactile examination, in that higher cut-off points were found in enamel than in dentine. This fact contributes to emphasize the lower reliability of the tested methods. ${ }^{24}$ Hence, tactile examination and radiography would not be the most appropriate methods to detect secondary caries at the dentine threshold, which disagrees with previous findings related to radiographic examination, ${ }^{7,11,25}$ but agrees with previous results related to tactile examination. ${ }^{26}$

Despite the limitation of the visual index used in detecting early secondary caries due to the difficulty in distinguishing discolorations originated from the restoration or from demineralizations, ${ }^{6}$ the visual inspection presented the highest sensitivity 
among the tested methods for both thresholds. On the other hand, some previous studies had shown that the LF performance was superior to that of the visual examination in detecting secondary caries lesions in permanent teeth. ${ }^{6,8}$ The scoring system used for the visual inspection aims at estimating lesion depth. ${ }^{14}$ The thinness of the primary enamel ${ }^{13}$ can explain the best performance in detecting secondary enamel lesions in primary teeth. The worse performance at the dentine threshold probably reflects the major difficulty in detecting dentinal caries not frankly cavitated.

Similar values of specificity for all methods at the enamel threshold mean that all methods performed similarly in identifying a sound site around an amalgam restoration. All methods presented specificity superior to 0.80 , which is a minimum value to assure a minimum false-positive fraction. ${ }^{11}$

The tactile examination generally presents high sensitivity and low specificity, resulting in a high number of false-positives. ${ }^{24}$ Nevertheless, in our study the tactile examination presented a combination of high specificity and low sensitivity. The gentle use of the explorer probe may have caused that. The probe was used without any pressure along the margins of the restoration in order to evaluate the presence of ditches and softened dental tissues. In spite of that, the other methods presented performances superior to that of the tactile examination, emphasizing that it is not the most indicated for detecting secondary caries lesions in primary teeth.

The low sensitivity and accuracy of radiography in detecting enamel secondary caries had already been expected because these lesions are rarely detected in bitewings radiographs. ${ }^{7,14,27}$ In addition, our study did not observe a good performance of this method at the dentine threshold, which does not corroborate the findings of other studies in which radiography presented acceptable values of sensitivity and specificity in the detection of advanced secondary caries. ${ }^{7,11}$ This divergence can be attributed to the presence of secondary lesions in the wall of the cavity, the presence of restorative material in the buccal or lingual surface or the occurrence of a shadow effect of the restorative material, ${ }^{28}$ which could make the detection of a demineralization related to sec- ondary caries in a bitewing radiograph difficult.

The visual inspection showed the highest accuracy in detecting secondary lesions in enamel. In dentine, the visual inspection was similar to LF in the detection of this kind of lesion. Thus, the suggestion of combining both methods in order to improve the detection of secondary caries lesions around amalgam restorations could be considered. This combination, however, did not increase the accuracy in detecting secondary caries around composites. ${ }^{8}$ Considering that this hypothesis has not been tested yet for amalgam restorations, future studies should be encouraged in order to verify that.

It is expected that objective methods should present higher agreement rates than subjective ones. Previous studies on the detection of secondary lesions have already demonstrated this claim. ${ }^{6}$ Nevertheless, the highest inter-examiner agreement in the present study was observed for the tactile examination, at the enamel threshold, and for the visual inspection, at the dentine threshold. The examiner's training in using visual and tactile criteria, ${ }^{29}$ the use of scoring systems and/or individual mistakes in operating the LF device ${ }^{30}$ can be possible explanations for this observation.

Considering the use of a single method to detect secondary caries lesions both in enamel and dentine, it would be recommendable that a method presenting high sensitivity for detecting initial (enamel) lesions and high specificity for detecting deep (dentine) lesions be chosen. Thus, it would be possible to adopt preventive measures as soon as possible and avoid the unnecessary replacement of restorations. ${ }^{6}$ In our study, the visual inspection combined both features with a high reliability, emphasizing its indication as the most effective method in detecting secondary caries lesions in primary teeth.

\section{Conclusion}

The visual inspection presented the best performance in detecting enamel and dentine secondary caries in primary teeth restored with amalgam.

\section{Acknowledgements}

The authors wish to thank Mr. Carlos Henrique Ferreira for his assistance with the English language. 


\section{References}

1. Kidd EA. Diagnosis of secondary caries. J Dent Educ. 2001;65(10):997-1000.

2. Elderton RJ. Clinical studies concerning re-restoration of teeth. Adv Dent Res. 1990;4:4-9.

3. Kidd EA, Toffenetti F, Mjor IA. Secondary caries. Int Dent J. 1992;42(3):127-38.

4. Kidd EA, Joyston-Bechal S, Beighton D. Marginal ditching and staining as a predictor of secondary caries around amalgam restorations: a clinical and microbiological study. J Dent Res. 1995;74(5):1206-11.

5. Gonzalez-Cabezas C, Fontana M, Gomes-Moosbauer D, Stookey GK. Early detection of secondary caries using quantitative, light-induced fluorescence. Oper Dent. 2003;28(4):41522.

6. Ando M, Gonzalez-Cabezas C, Isaacs RL, Eckert GJ, Stookey GK. Evaluation of several techniques for the detection of secondary caries adjacent to amalgam restorations. Caries Res. 2004;38(4):350-6.

7. Bamzahim M, Shi XQ, Angmar-Mansson B. Secondary caries detection by DIAGNOdent and radiography: a comparative in vitro study. Acta Odontol Scand. 2004;62(1):61-4.

8. Boston DW. Initial in vitro evaluation of DIAGNOdent for detecting secondary carious lesions associated with resin composite restorations. Quintessence Int. 2003;34(2):109-16.

9. Hibst R, Paulus R, Lussi A. Detection of occlusal caries by laser fluorescence: Basic and Clinical investigations. Med Laser Appl. 2001;16:205-13.

10. Bader JD, Shugars DA. A systematic review of the performance of a laser fluorescence device for detecting caries. J Am Dent Assoc (1939). 2004;135(10):1413-26.

11. Bamzahim M, Aljehani A, Shi XQ. Clinical performance of DIAGnodent in the detection of secondary carious lesions. Acta Odontol Scand. 2005;63(1):26-30.

12. Boston DW, Sauble JE. Evaluation of laser fluorescence for differentiating caries dye-stainable versus caries dye-unstainable dentin in carious lesions. Am J Dent. 2005;18(6):351-4.

13. Mortimer KV. The relationship of deciduous enamel structure to dental disease. Caries Res. 1970;4(3):206-23.

14. Ekstrand KR, Ricketts DNJ, Kidd EAM. Reproducibility and accuracy of three methods for assessment of demineralization depth of the occlusal surface: an in vitro examination. Caries Res. 1997;31(3):224-31.

15. Mendes FM, Hissadomi M, Imparato JCP. Effects of drying time and the presence of plaque on the in vitro performance of laser fluorescence in occlusal caries of primary teeth. Caries Res. 2004;38(2):104-8.

16. Yazici AR, Baseren M, Gokalp S. The in vitro performance of laser fluorescence and caries-detector dye for detecting residual carious dentin during tooth preparation. Quintessence Int. 2005;36(6):417-22.
17. Lussi A, Imwinkelried S, Pitts N, Longbottom C, Reich E. Performance and reproducibility of a laser fluorescence system for detection of occlusal caries in vitro. Caries Res. 1999;33(4):261-6.

18. El-Housseiny AA, Jamjoum H. Evaluation of visual, explorer, and a laser device for detection of early occlusal caries. J Clin Pediatr Dent. 2001;26(1):41-8.

19. Fung L, Smales R, Ngo H, Moun G. Diagnostic comparison of three groups of examiners using visual and laser fluorescence methods to detect occlusal caries in vitro. Aust Dent J. 2004;49(2):67-71.

20. Baseren NM, Gokalp S. Validity of a laser fluorescence system (DIAGNOdent) for detection of occlusal caries in third molars: an in vitro study. J Oral Rehabil. 2003;30(12):1190-4.

21. Reis A, Mendes FM, Angnes V, Angnes G, Grande RHM, Loguercio AD. Performance of methods of occlusal caries detection in permanent teeth under clinical and laboratory conditions. J Dent. 2006;34(2):89-96.

22. Francescut P, Lussi A. Correlation between fissure discoloration, Diagnodent measurements, and caries depth: an in vitro study. Pediatr Dent. 2003;25(6):559-64.

23. Francescut P, Zimmerli B, Lussi A. Influence of different storage methods on laser fluorescence values: a two-year study. Caries Res. 2006;40(3):181-5.

24. Bader JD, Shugars DA, Bonito AJ. A systematic review of the performance of methods for identifying carious lesions. J Public Health Dent. 2002;62(4):201-13.

25. Rudolphy MP, van Loveren C, van Amerongen JP. Grey discoloration for the diagnosis of secondary caries in teeth with class II amalgam restorations: an in vitro study. Caries Res. 1996;30(3):189-93.

26. Rudolphy MP, van Amerongen JP, Penning C, ten Cate JM. Grey discolouration and marginal fracture for the diagnosis of secondary caries in molars with occlusal amalgam restorations: an in vitro study. Caries Res. 1995;29(5):371-6.

27. Rocha RO, Ardenghi TM, Oliveira LB, Rodrigues CRMD, Ciamponi AL. In vivo Effectiveness of Laser Fluorescence Compared to Visual Inspection and Radiography for the Detection of Occlusal Caries in Primary Teeth. Caries Res. 2003;37(6):437-41.

28. Espelid I, Tveit AB, Erickson RL, Keck SC, Glasspoole EA. Radiopacity of restorations and detection of secondary caries. Dent Mater. 1991;7(2):114-7.

29. Verdonschot EH, Angmar-Mansson B, ten Bosch JJ, Deery $\mathrm{CH}$, Huysmans MC, Pitts NB et al. Developments in caries diagnosis and their relationship to treatment decisions and quality of care. ORCA Saturday Afternoon Symposium 1997. Caries Res. 1999;33(1):32-40.

30. Kuhnisch J, Ziehe A, Brandstadt A, Heinrich-Weltzien R. An in vitro study of the reliability of DIAGNOdent measurements. J Oral Rehabil. 2004;31(9):895-9. 